\title{
Evaluación de constructos relacionados con las Teorías del Aprendizaje Social-Cognitivo en drogodependientes en tratamiento: fiabilidad y validez
}

\author{
Cesáreo Fernández Gómez*; Juan Miguel Llorente del Pozo** \\ * Centro para la Investigación y Desarrollo en Comunidad Terapéutica (CIDECOT) \\ * Servicio Vasco de Salud IOSAKIDETZA \\ Enviar correspondencia a: \\ Cesáreo Fernández Gómez. B Samese, 52 A. 48114 Arrieta. Vizcaya. España. cesareofg@cidecot.org
}

Recibido: 5 de abril de 2005. Aceptado: 3 de abril de 2006.

\section{RESUMEN}

El estudio realiza una primera aproximación para la validación del modelo basado en el Aprendizaje Social - Cognitivo en el tratamiento de deshabituación a opiáceos. Se ha utilizado una batería de cuestionarios para la evaluación de los constructos incluidos en el modelo: Situaciones en las que se consumen drogas, Atribuciones Causales del consumo y la abstinencia, Expectativa del lugar de control sobre el consumo de drogas, Expectativas de Resultados de la abstinencia, Estadios de Cambio en la conducta adictiva y Autoeficacia. La muestra incluye pacientes politoxicómanos dependientes a opiáceos en diferentes programas de tratamiento de todo el Estado $(N=671)$. Los instrumentos de evaluación utilizados han mostrado una consistencia interna satisfactoria, estimada mediante el coeficiente alfa de Cronbach, con el $62 \%$ de estos coeficientes superiores a 0,80 y el $25 \%$ restante superior a 0,70 . Se encuentra una validez de constructo adecuada (convergente y divergente), con un análisis factorial exploratorio que apoya la validez de los constructos utilizados por la Teoría del Aprendizaje Social - Cognitivo, agrupando en cuatro factores: 1) los constructos relativos a atribuciones causales, 2) la situación psicológica y motivación al cambio, 3) resultados esperados de la abstinencia y su valor subjetivo, y 4) autoeficacia y lugar de control sobre el consumo de drogas. Los instrumentos de evaluación estudiados pueden resultar útiles para la planificación y evaluación de intervenciones psicológicas en drogodependientes.

Palabras clave: comunidad terapéutica, evaluación, tratamiento, aprendizaje, social-cognitivo

\begin{abstract}
This study provides a first approach on validating the Social Learning Theory for drug abuse treatment (heroin). We used a set of questionnaires designed to measure different constructs related to Social Learning Theory: Drug use situations, Causal dimensions for drug use and abstinence, Locus of control related to drug use, Outcome expectancies for abstinence, Stages of change and Self-efficacy. Sample includes 671 polidrug opiate-dependent patients was assessed. The assessment instruments showed an adequate internal consistency, with $62 \%$ of constructs showing an alpha coefficient higher than 0.80 and other $25 \%$ coefficients higher than 0.70 . We also found satisfactory construct validity (convergent and divergent), with an exploratory factor analysis providing some support to the constructs included in the original model, by grouping the explored variables in four factors: 1) causal dimensions related constructs, 2) psychological situation and motivation for change; 3) outcome expectancies for abstinence and their subjective value, 4) self-efficacy and locus of control related to drug use. The studied assessment instruments can be useful in planning and assessing psychological interventions for drug-dependent individuals
\end{abstract}

Key words: Therapeutic community, assessment, treatment, learning, social-cognitive

\section{INTRODUCCIÓN}

Las teorías del Aprendizaje Social nacen con la formulación de Rotter (1954), en un esfuerzo por integrar las teorías de $\mathrm{E}-\mathrm{R}$ (estímulo - respuesta, o teorías del reforzamiento) y las teorías de campo o "cognitivas", y fueron desarrolladas para la predicción de la conducta humana en situaciones sociales complejas rechazando el determinismo (Pérez, 1986). Esta teoría estudia la conducta como un resultado de interacción entre la persona y su entorno, desarrolla una descripción detallada de la situación psicológica del individuo y las condiciones ambientales (metas o reforzamientos) y realiza una aproximación histórica al estudio de la per- 
sonalidad, con énfasis especial en las necesidades individuales y las "expectativas" que tiene la persona de que esas metas serán alcanzadas y esas necesidades satisfechas. En términos operativos, la probabilidad de una conducta en relación con otras alternativas ante una determinada situación psicológica vendrá determinada por las expectativas del individuo y el valor de los refuerzos esperados y alcanzados. Las expectativas son las probabilidades que ve el individuo (subjetivas) de que un refuerzo o meta particular ocurra como una función o resultado de una conducta específica propia ante tal determinada situación. Las expectativas consideradas en la teoría incluyen las expectativas de reforzamiento (expectativas de resultados contingentes a una conducta), las expectativas de solución de problemas (autoeficacia) y las expectativas de control sobre los refuerzos (dimensión de control interno - externo o "locus de control"). El valor del refuerzo es el grado de preferencia que la persona muestra por un determinado refuerzo o meta, y se define operativamente por el gradiente de reforzamiento de un sujeto (preferencias) o por la elección conductual (elección entre diversas alternativas cuando todas ellas son igualmente posibles). Finalmente, la situación psicológica hace referencia a cómo el individuo percibe y valora su situación, definida como un conjunto complejo de claves (conductas y acontecimientos inmediatos o previos) que actúan sobre el individuo y que determinan las expectativas de reforzamiento y los valores de los reforzadores. Una formulación más reciente de la teoría del Aprendizaje Social se encuentra en la extensa obra de Bandura (1999), que ha sido desarrollada y utilizada como acercamiento social - cognitivo (Becoña, 1999). En esta teoría de Bandura cobra gran importancia el concepto de autoeficacia como constructo explicativo de la conducta humana.

Las teorías basadas en el Aprendizaje Social - Cognitivo han sido aplicadas a la explicación integradora del consumo de sustancias psicoactivas, sirviendo de base para el desarrollo de intervenciones orientadas a la prevención de recaídas en la deshabituación en la conducta adictiva (Llorente, 1997). Básicamente, las intervenciones basadas en estas teorías consideran que las intervenciones para la deshabituación de sustancias psicoactivas tienen más probabilidad de éxito si aumentan en el sujeto: (1) la ubicación en una situación psicológica favorable al cambio y extinción de la conducta adictiva, (2) las expectativas de control sobre las conductas necesarias para alcanzar dichos cambios, (3) las expectativas de resistir los deseos de consumir sustancias de abuso, (4) las expectativas de resultados positivos contingentes al cambio y (5) la valoración positiva de las consecuencias y resultados contingentes a este cambio en la conducta adictiva.

Las teorías del aprendizaje Social han dado lugar a una extensa literatura científica relativa al tratamien- to en la conducta adictiva, y en menor medida en el consumo de opiáceos. La mayoría de estas investigaciones se han orientado hacia aspectos parciales, han estudiado por separado las principales variables explicativas incluidas en el modelo y la mayoría de estas investigaciones se han orientado hacia el estudio de las expectativas de autoeficacia (Llorente y Fernández, 1999). En menor medida se han estudiado la situación psicológica experimentada por el sujeto, las expectativas de control sobre las conductas necesarias para el cambio de la conducta adictiva, las expectativas de resultados de dicho cambio y la valoración subjetiva de tales resultados esperados o alcanzados tras dicho cambio comportamental. El objetivo general de este trabajo es estimar las propiedades psicométricas de una serie de instrumentos disponibles en español para evaluar los principales constructos integrados en este modelo, en una muestra amplia de drogodependientes en tratamiento. Los objetivos específicos son estimar la fiabilidad de estos instrumentos 8consistencia interna) y su validez (convergente y divergente).

\section{MATERIAL Y MÉTODO}

\section{Muestra}

Los sujetos participantes en el estudio se encontraban siguiendo tratamiento por dependencia de opiáceos en programas distribuidos por el Estado Español. El tamaño total de la muestra es de 671 sujetos, la mayoría son hombres $(82,8 \%)$, con una media de edad de 28,6 años (D.E. 4,7), están solteros (71,9\%) y viven con la familia de origen (72,5\%); el 17,5\% están casados o tienen pareja estable y el $24,4 \%$ tiene hijos. El nivel educativo más frecuente es bajo, sin Graduado Escolar (36,9\%); y la situación laboral más frecuente es el desempleo sin percepción de prestaciones $(56,8 \%)$. No se observan diferencias entre la muestra del presente estudio y la descrita en el informe del Sistema Estatal de Información en Toxicomanías (S.E.I.T.) referida a los admitidos a tratamiento en cuanto a la proporción de hombres y mujeres (prueba Binomial: $p=0,2782$, para el contraste unilateral; $\chi 2$; $p=0$,4327). Tampoco se observan diferencias entre ambas muestras en cuanto a la edad $(\mathrm{t}=0,48 ; 658$ g.l.; $p=0,634$ ). Son consumidores habituales (consumían al menos varias veces por semana) de heroína desde hace 8,4 años (D.E. 4,3 años), En el momento de la evaluación, la mayoría de los sujetos se encontraban en tratamiento en programas de comunidad terapéutica $(81,1 \%)$, mientras el resto se encontraban en tratamiento en programas de desintoxicación, terapia ambulatoria y programas de agonistas y antagonistas opiáceos. Todos los sujetos participantes en el estudio cumplían los criterios de dependencia de opiáceos (304.0), según el DSM-IV. 


\section{Instrumentos}

Se utilizó una batería de cuestionarios auto-administrados para la evaluación de los constructos de las Teorías del Aprendizaje Social - Cognitivo:

El Inventario de Situaciones en las que se Consumen Drogas (Inventory of Drug Taking Situations - IDTS) es un cuestionario autoadministrado de 50 ítems desarrollado por Annis y Graham (1992) para identificar las situaciones en las que un paciente adicto consumió drogas en el último año. Las situaciones evaluadas por el IDTS se basan en la clasificación de 8 categorías llevada a cabo por Marlatt y colaboradores: estados emocionales desagradables, malestar fisiológico, estados emocionales agradables, querer probar el control personal sobre la sustancia, experimentación de fuertes deseos y tentaciones de consumo, presión social, conflictos interpersonales y estados emocionales agradables en compañía de otros. Para representar convenientemente cada categoría, los autores Annis y Graham (1992) generaron un total de 50 ítems que se agrupan en 8 subescalas. Como resultado de la corrección, se obtienen 8 puntuaciones que proporcionan el Perfil de Situaciones de Riesgo para el consumo de drogas.

Escala de Atribuciones Causales. "Causal Dimension Scale" (CDS II), desarrollado por McAuley, Duncan y Russell (1992). La "Causal Dimension Scale II" fue desarrollada con el fin de evaluar las atribuciones causales que hace una persona de los éxitos y/o fracasos que tiene en su vida: localización (interna o externa), estabilidad, controlabilidad interna y controlabilidad externa. La CDS II que hemos aplicado es, a su vez, la versión utilizada por Vielva (1994) adaptada a la muestra de heroína. Para ello, se sustituyeron las alusiones al consumo de alcohol por referencias al consumo de heroína y a la abstinencia de heroína. De este modo se evalúan las cuatro dimensiones causales mencionadas en relación con la abstinencia y recaída en el consumo de heroína.

Escala de Expectativa del Lugar de Control: La escala empleada por Annis, Sklar y Turner (1996) para evaluar las expectativas de lugar de control que tienen los drogodependientes sobre el abandono de las drogas, está compuesta por 25 ítems y su formato de respuestas es la elección forzosa de una de las dos alternativas que se presentan en cada ítem: una alternativa representa la creencia de externalidad y la otra alternativa representa la creencia de internalidad. Se pregunta a los sujetos por sus expectativas de control sobre el dejar de consumir heroína. Debido a su aplicación a la adicción a la heroína, la escala utilizada recibe el nombre de Drug I-E o “Expectativas de lugar de control (interno-externo) sobre la adicción a las drogas".

Escala de Expectativas de Resultados: El cuestionario utilizado para medir las expectativas de resulta- do en pacientes heroinómanos que están tratando de dejar la heroína se deriva del desarrollado por Solomon y Annis (1989) para población alcohólica: el Outcome Expectancy Scale (OES). Al igual que lo realizado por Annis, Sklar y Turner (1996) en este estudio se ha procedido a adaptar la escala a la problemática de la adicción a la heroína. La escala está formada por 34 ítems que expresan las consecuencias posibles (positivas y negativas) que se pueden esperar tras abandonar el consumo de drogas. El sujeto debe contestar si le gustaría que le ocurrieran esas consecuencias y en qué medida cree que le van a ocurrir a él. Dicha escala presenta, por lo tanto, dos partes o subescalas: 1) Una primera subescala, Ilamada Valencia, que hace referencia al valor y la importancia que el sujeto adjudica a cada una de las 34 consecuencias. 2) Una segunda subescala, llamada Fuerza, que se refiere al grado en que el sujeto cree que cada una de esas consecuencias le ocurrirán si abandona las drogas. La inclusión de estos dos componentes se debe a que tanto la percepción de que tendrá lugar una determinada consecuencia como su valor o importancia subjetiva influyen en la conducta que adopte el heroinómano.

Cuestionario para la evaluación de la situación psicológica en relación con el cambio en la conducta adictiva: Cuestionario para la Evaluación de los Estadios de Cambio (SOCRATES). El Stages of Change Readiness and Treatment Eagerness Scale (SOCRATES, Miller y Rollnick, 1991) es un instrumento experimental diseñado para evaluar la preparación para el cambio en sujetos con problemas de abuso o dependencia del alcohol u otras sustancias. El instrumento proporciona puntuaciones en cinco escalas derivadas racionalmente, que corresponden a los cinco estadios de cambio conceptualmente considerados por Prochaska y DiClemente, en el Modelo Transteórico del cambio en las conductas adictivas. Las cinco escalas o estadios de cambio evaluados son: Precontemplación (P), Contemplación (C), Determinación/Decisión (D), Acción (A) y Mantenimiento (M).

Cuestionario de Confianza en Situaciones de Riesgo para Consumir Drogas. El Drug Taking Confidence Questionnaire (DTCQ), desarrollado por Annis y Martin (1985) es un cuestionario autoaplicado de 50 ítems, diseñado para evaluar autoeficacia (o confianza) en la propia capacidad de resistir al deseo de utilizar drogas en diferentes tipos de situaciones. Las situaciones de consumo de drogas o alcohol evaluadas mediante el DTCQ están basadas en el sistema de clasificación de 8 categorías desarrollado por Marlatt y colaboradores. Mediante el DTCQ se obtienen puntuaciones en ocho subescalas; cinco de ellas se refieren a situaciones Personales o Intrapersonales, en las cuales el uso de sustancias implica una respuesta a un evento que es principalmente de naturaleza psicológica o física. Estas cinco subescalas son denominadas: [Emociones Desagradables: 10 ítems; Malestar Físico: 5 
ítems; Emociones Agradables: 5 ítems; Pruebas de Control Personal: 5 ítems; y Urgencias y Tentaciones: 5 ítems]. Las tres subescalas restantes se refieren a situaciones que implican a otras personas, o situaciones Interpersonales, en las cuales está implicada una influencia significativa de parte de otro individuo o individuos: [Conflicto con Otros: 10 ítems; Presión Social hacia el Consumo: 5 ítems; y Momentos Agradables con Otros - Celebraciones: 5 ítems]. Las puntuaciones en las ocho subescalas constituyen un perfil de confianza del sujeto, mostrando el nivel de confianza percibido por el individuo, a través de los ocho tipos de situaciones de consumo de sustancias.

\section{Procedimientos}

Los análisis estadísticos se realizaron con el paquete estadístico SPSS. Para determinar la consistencia interna en cada una de las escalas y subescalas se ha utilizado el coeficiente alfa de Cronbach y la correlación media inter-ítems en cada escala. Para evaluar la asociación entre las diferentes escalas se aplicó un análisis factorial de componentes principales con una rotación Oblimin para la rotación e identificación de la solución factorial elegida.

Tabla 1: Indicadores de fiabilidad (consistencia interna) de los instrumentos utilizados para la evaluación de las variables incluidas en la Teoría del Aprendizaje Social - Cognitivo.

\begin{tabular}{|c|c|c|c|c|}
\hline \multicolumn{2}{|c|}{ ESCALA } & ÍTEMS & R. MEDIA & A \\
\hline \multirow{8}{*}{$\begin{array}{r}\text { Inventory of } \\
\text { Drug Taking } \\
\text { Situations } \\
\text { (IDTS) }\end{array}$} & Emociones Desagradables & 10 & 0,53 & 0,92 \\
\hline & Malestar Físico & 5 & 0,37 & 0,75 \\
\hline & Emociones Agradables & 5 & 0,61 & 0,89 \\
\hline & Intentos de Control & 5 & 0,56 & 0,87 \\
\hline & Tentaciones / Urgencias & 5 & 0,41 & 0,78 \\
\hline & Conflictos con Otros & 10 & 0,55 & 0,92 \\
\hline & Presión Social & 5 & 0,53 & 0,85 \\
\hline & Momentos Agradables con Otros & 5 & 0,52 & 0,84 \\
\hline \multirow{8}{*}{$\begin{array}{r}\text { Causal } \\
\text { Dimension } \\
\text { Scale } \\
\text { (CDS-II) }\end{array}$} & Abstinencia - Internalidad & 3 & 0,53 & 0,77 \\
\hline & Abstinencia - Control Interno & 3 & 0,38 & 0,65 \\
\hline & Abstinencia - Estabilidad & 3 & 0,29 & 0,55 \\
\hline & Abstinencia - Control Externo & 3 & 0,59 & 0,81 \\
\hline & Recaída - Internalidad & 3 & 0,63 & 0,84 \\
\hline & Recaída - Control Interno & 3 & 0,45 & 0,71 \\
\hline & Recaída - Estabilidad & 3 & 0,46 & 0,72 \\
\hline & Recaída - Control Externo & 3 & 0,65 & 0,85 \\
\hline \multicolumn{2}{|c|}{ Drug Related I-E Locus of Control (DRIE) } & 25 & 0,12 & 0,78 \\
\hline \multirow{6}{*}{$\begin{array}{r}\text { Outcome } \\
\text { Expectancy } \\
\text { Scale } \\
\text { (OES) }\end{array}$} & Beneficios (Valor) & 21 & 0,31 & 0,90 \\
\hline & Costes (Valor) & 13 & 0,24 & 0,80 \\
\hline & Beneficios (Fuerza) & 21 & 0,44 & 0,94 \\
\hline & Costes (Fuerza) & 13 & 0,23 & 0,79 \\
\hline & Beneficios (Expectativas) & 21 & 0,44 & 0,94 \\
\hline & Costes (Expectativas) & 13 & 0,21 & 0,77 \\
\hline \multirow{5}{*}{ SOCRATES } & Precontemplación & 8 & 0,17 & 0,62 \\
\hline & Contemplación & 8 & 0,13 & 0,55 \\
\hline & Determinación & 8 & 0,27 & 0,74 \\
\hline & Actuación & 8 & 0,39 & 0,84 \\
\hline & Mantenimiento & 8 & 0,23 & 0,70 \\
\hline \multirow{9}{*}{$\begin{array}{r}\text { Drug } \\
\text { Taking } \\
\text { Confidence } \\
\text { Questionnaire } \\
-\mathrm{H}\end{array}$} & Emociones Desagradables & 10 & 0,57 & 0,93 \\
\hline & Malestar Físico & 5 & 0,47 & 0,81 \\
\hline & Emociones Agradables & 5 & 0,60 & 0,88 \\
\hline & Intentos de Control & 5 & 0,62 & 0,89 \\
\hline & Tentaciones / Urgencias & 5 & 0,49 & 0,83 \\
\hline & Conflictos con Otros & 10 & 0,57 & 0,93 \\
\hline & Presión Social & 5 & 0,64 & 0,90 \\
\hline & Momentos Agradables con Otros & 5 & 0,54 & 0,85 \\
\hline & DTCQ-H Total & 50 & 0,44 & 0,97 \\
\hline
\end{tabular}




\section{RESULTADOS}

Este apartado está estructurado de la siguiente forma: En primer lugar se muestran los resultados relativos a la fiabilidad (consistencia interna) de las medidas de las variables incluidas en la Teoría del Aprendizaje Social - Cognitivo. Posteriormente se incluyen los resultados relativos a la validez de constructo (convergente y divergente) de las variables incluidas en dichos modelos,

\section{Evaluación de la fiabilidad (consistencia inter- na) de los instrumentos seleccionados}

Los resultados recogidos en la tercera columna de la tabla 1 muestran como el coeficiente alfa es superior a 0,70 en casi todas las escalas, con la excepción de algunas subescalas relativas a la situación psicológica (estadios de Precontemplación y Contemplación) y algunas escalas de los estilos atributivos que están formadas por muy pocos ítems (Estabilidad y Control Interno de la abstinencia de heroína), en las que este valor se aproxima o alcanza el valor de 0,60. El coeficiente alfa como indicador de la consistencia interna alcanza valores superiores a 0,80 en la mayoría de las escalas. Los coeficientes de correlación media entre los ítems de cada escala están por encima de 0,30 también en la mayoría de ellas. En resumen, estos resultados indican que las variables incluidas en la Teoría del Aprendizaje Social - Cognitivo pueden ser evaluados con los instrumentos utilizados en el presente estudio, obteniendo medidas relativamente fiables de las variables que se pretenden estudiar.

\section{Evaluación de la validez de constructo (conver- gente $y$ divergente)}

La validez de constructo se refiere a las variables que deberían asociarse positivamente (validez convergente) y las que no deberían asociarse substancialmente (validez divergente). Con el fin de esclarecer estas asociaciones, en la tabla 2 se muestran los resultados del análisis factorial de componentes principales. Se eligió una solución de 4 factores que explica algo más de la mitad de la varianza de las 23 variables $(51,4 \%)$, tras rechazar la solución inicial de 7 factores por resultar los tres últimos difícilmente interpretables. Cinco de las 23 variables no alcanzaron un peso superior a 0,40 en ningún factor: La estabilidad de las causas de la abstinencia y de la recaída, el estadio de Contemplación, el valor subjetivo de los resultados negativos de la abstinencia, la importancia de la abstinencia y la puntuación total en el IDTS.

Tabla 2: Solución factorial obtenida con las variables incluidas en el modelo del Aprendizaje Social - Cognitivo.

\begin{tabular}{|l|l|l|l|}
\hline \multicolumn{1}{|c|}{ FACTOR 1 } & \multicolumn{1}{c|}{ FACTOR 2 } & \multicolumn{1}{c|}{ FACTOR 3 } & \multicolumn{1}{c|}{ FACTOR 4 } \\
\hline Recaída Interna $(0,72)$ & Acción $(0,75)$ & Expectativas & Expectativas \\
Abstinencia Interna $(0,70)$ & Precontemplación $(-0,75)$ & positivas $(0,94)$ & negativas $(-0,81)$ \\
Abstinencia control interno $(0,62)$ & Determinación $(0,74)$ & $\begin{array}{l}\text { Balance decisional } \\
\text { abstinencia }(0,89)\end{array}$ & Costes de la \\
abstinencia $(-0,80)$ \\
Control Externo - Recaída $(0,61)$ & Mantenimiento $(0,67)$ & $\begin{array}{l}\text { Probabilidad de los } \\
\text { resultados positivos }(0,89)\end{array}$ & Autoeficacia $(0,65)$ \\
Control Interno - Recaída $(0,58)$ & & Valor de los resultados & Locus de \\
Control Externo - Abstinencia $(0,57)$ & & positivos $(0,68)$ & \\
Locus de Control Interno $(0,53)$ & & & \\
\hline
\end{tabular}

El primer factor parece asociarse claramente con los estilos atributivos en la abstinencia y consumo de heroína. El segundo factor parece representar muy aceptablemente los conceptos relativos a la situación psicológica relativa a la conducta en cuestión (el cambio en la conducta adictiva relacionada con la heroína). El tercer factor está asociado notablemente al factor relativo a los resultados esperados de la abstinencia y al valor subjetivo de los mismos. El cuarto factor se asocia a las expectativas de Autoeficacia y el Locus de
Control Interno, los cuales se asocian fuertemente a la falta de expectativas negativas y costes de la abstinencia.

\section{DISCUSIÓNY CONCLUSIONES}

El estudio es un intento de proporcionar instrumentos para enfocar el proceso de deshabituación a drogas 
integrando los principales constructos del Aprendizaje Social Cognitivo, haciendo especial énfasis en su fiabilidad y validez. La utilidad del estudio sería máxima si sus resultados fuesen generalizables. El diseño aplicado en este estudio es diferente del clásico método de encuestas, ya que no se emplea el muestreo aleatorio de la población española de dependientes a heroína en tratamiento. Sin embargo, la variedad geográfica de los sujetos y el hecho de que la muestra del estudio presente características comparables en sexo, edad y duración del consumo de heroína con la población española de referencia, aumenta la posible validez de los resultados.

El objetivo principal es determinar la fiabilidad y validez de las medidas utilizadas para evaluar los constructos relacionados con el Aprendizaje Social Cognitivo en población española. La estimación de la fiabilidad basada en la consistencia interna se interesa por la homogeneidad de los ítems que forman una determinada escala, es decir, por la medida en que los ítems que la forman miden un mismo constructo (DeVellis, 1991). Generalmente se acepta que el valor del coeficiente $\nabla$ no debe ser nunca menor de 0,70 (Nunnally, 1978). Dos de las escalas con un coeficiente inferior a ese valor están formadas solamente por 3 ítems, y este resultado es esperable porque se reconoce que este coeficiente de consistencia interna aumenta con el número de ítems (Ebel, 1972). Una correlación entre ítems igual o superior a 0,20 aumenta la fiabilidad de la escala (Nunnally, 1978), y se observa que casi la totalidad de las escalas utilizadas en el estudio alcanzan una correlación media entre ítems superior a ese valor (el 90\%). Nuestros resultados en general demuestran una consistencia interna elevada, indicando que los sujetos heroinómanos evaluados son consistentes a través de los diferentes ítems que forman cada una de las escalas y subescalas utilizadas.

La validez de constructo es el aspecto fundamental de la validez de las escalas que pretenden estimar los conceptos latentes definidos por la Teoría del Aprendizaje Social (Messick, 1980). Este tipo de validez estudia la relación teórica y empírica entre las variables que componen este modelo teórico del Aprendizaje Social - Cognitivo, en el marco denominado "red nomológica" (Cronbach y Meehl, 1955; DeVellis, 1991). Esta "red" de asociaciones los constructos integrados en el Aprendizaje Social - Cognitivo en conductas adictivas está en su primera fase de desarrollo, existiendo algunas hipótesis que relacionan la autoeficacia con otras variables (Llorente, 1997). Por ello, el objetivo es explorar empíricamente algunas asociaciones entre estas variables, más que contrastar unas relaciones ya han verificadas en otros estudios. En general, la dirección de las relaciones entre constructos se ajusta a lo predecible desde la Teoría del Aprendiza- je Social - Cognitivo y al mismo tiempo la magnitud de las asociaciones encontradas apoya firmemente la independencia de las variables medidas. Por tanto, los resultados sobre la interrelación entre las variables incluidas en el modelo apoyan la validez global del modelo y la validez de constructo de las variables tal y como han sido evaluadas en el presente estudio. En conclusión, los instrumentos adaptados para evaluar los constructos integrados en la Teoría del Aprendizaje Social - Cognitivo han demostrado fiabilidad y validez de constructo aceptables. Estos instrumentos pueden ser útiles para la planificación y evaluación de intervenciones basadas en esta teorías en drogodependientes en deshabituación.

\section{AGRADECIMIENTOS:}

Este estudio fue premiado con el premio Zambón - Socidrogalcohol 2000.

\section{REFERENCIAS}

Annis, H.M.; Martin, G. (1985). Drug-Taking Confidence Questionnaire. Toronto: Addiction Research Foundation.

Annis, H.M.; Graham, J.M. (1992) Inventory of Drugtaking Situations: (IDTS) User's Guide, Adolescent Version. Toronto: Addiction Research Foundation.

Annis, H.M.; Sklar, S.M.; Turner, N.E. (1996). DrugTaking Confidence Questionnaire (DTCQ) User's Guide. Addiction Research Foundation. Toronto.

Bandura, A. (1999). A sociocognitive analysis of substance abuse: An agentic perspective. Psychological Science, 10, 214-217.

Becoña, E. (1999). Bases teóricas que sustentan los programas de prevención de drogas. Madrid: Plan Nacional Sobre Drogas.

Cronbach, L. J.; Meehl, P.E. (1955). Construct validity in psychological tests. Psychological Bulletin, 52, 281302.

Devellis, R.F. (1991). Scale Development: Theory and Applications. London. Sage Publications.

Ebel, R. (1972). Why is a longer test usually a more reliable test?. Educational and psychological Measurement, 32, 249-253.

Llorente, J. (1997). Validación del DTCQ-H en población española. Tesis doctoral no publicada. Madrid: Universidad Nacional de Educación a Distancia (UNED).

Llorente, J.m.; Fernández, C. (1999). La autoeficacia y su relación con la abstinencia y recaída en el proceso de deshabituación de drogas. En: Becoña, E.; Rodríguez, A.; Salazar, I.; (Eds.): Drogodependencias, Vol. No 5. Universidad de Santiago de Compostela. 209-248.

Mcauley, E.; Duncan, T.e.; Russell, D.w. (1992). Measuring causal attributions: The revised Causal Dimension Scale 
(CDS II). Personality and Social Psychology Bulletin, 18, 566-573.

Messick, S. (1980). Test validity and the ethics of assessment. American Psychologist, 35, 11, 1012-1027.

Miller, W.R.; ROLLNICK, S. (1991). Motivational interviewing. Preparing people to change addictive behavior. New York: Guilford Press.

Nunnally, J.C. (1978). Psychometric Theory. New York: McGraw-Hill.

Pérez garcía, A.M. (1986). Aprendizaje social y personalidad: el modelo de J.B. Rotter. En: BERMÚDEZ. J.: Psicología de la Personalidad. Madrid: Universidad nacional de Educación a distancia (UNED).

Rotter, J.b. (1954). Social learning and clinical psychology. Englewood Cliffs, N.J.: Prentice-Hall.

Solomon, K.e.; Annis, H.m. (1989). Development of a Scale to Measure Outcome Expectancy in alcoholics. Cognitive Therapy and Research, 13, 409-421.

Vielva, I. (1994). Percepción de control y afrontamiento en el proceso de rehabilitación del alcohólico. Tesis Doctoral. Universidad de Deusto. 
